\title{
THE TREATMENT OF THE DESPERATE GOITER PATIENT*
}

\author{
J. EARL ELSE, M.D.
}

PORTLAND, OREGON.

T HE mortality and morbidity following operations for toxic goiter are now fairly good in patients with moderately toxic goiter showing no extraordinary symptoms or pathology. The great difference between the mortality and morbidity rate of surgeons obtaining the best results and those obtaining only mediocre results is usually in the so-called desperate goiter patients. This paper will discuss the following: $I$, severe hyperthyroidism without crisis; 2, hyperthyroidism with crisis; 3 , hyperthyroidism with fibrillation; 4, hyperthyroidism with extreme hypertension; 5, hyperthyroidism in pregnancy.

I. Severe hyperthyroidism without crisis. It would seem that with the use of Lugol's solution as suggested by Plummer and of the preparation of the patient for operation as emphasized by Crile, the mortality should be low, yet it is not as low as it should be. The chief cause of mortality and morbidity in this group is lack of preparation before operation. The patients are operated upon before they are ready and death is the result. The master goiter surgeon may be able to judge when the patient is ready for operation without laboratory assistance, but the mass of surgeons operating upon goiter cannot, and death often results in their attempt to imitate the master by disregarding such aids. While the master goiter surgeon by his skill may be able to tell when the severely toxic patient is ready for operation, he cannot by his skill so accurately distinguish between the mildly toxic goiter and other conditions which so closely simulate it that only the basal metabolic rate will differentiate. Neither can he by his skill so accurately distinguish between the patient requiring stage operations and the one who does not, so he treats all as severe cases. There is no surgeon so skilled that he can differentiate as well without laboratory aids as with them; and the surgeon who does not use laboratory aids either subjects some of his patients to unnecessary delay with unnecessary stage operations, or has an operative death-rate higher than it should be.

Patients with severe hyperthyroidism should be sent to the hospital for preparation. It cannot be done well at home. Patients should not be permitted to get out of bed for anything or consulted about any matters pertaining to business or other responsibilities. Lugol's solution should be given in amounts varying from Io minims three times a day to 25 minims four times a day according to the severity of the disease. Nervousness should be controlled by giving 5 to 10 grains of veronal in hot milk every night at 90 'clock as routine, and additional veronal or pantopon if needed to make the patient perfectly contented. The diet should be watched, with selection of food that the patient likes and can digest easily, and eggnog, milk or chocolate should be given between meals. We make it an absolute rule never to do a radical operation on such a patient with a basal metabolic rate over plus $5^{\circ}$; under plus 40 is preferable and even then we do not operate untiI the patient's symptoms show a very definite improvement. Further, it is an absolute rule never to operate upon such a patient if he is apprehensive of the result. I do not believe in the so-called stealing of the goiter. With the judicious use of sedatives, Lugol's solution, a carefully forced diet * Read before the American Association for the Study of Goiter, Denver, June I8-20, 1928. 
and proper nursing, these patients instead of being apprehensive are anxious for the day of operation to come. In the occasional patient who cannot be satisfactorily prepared by this method, the stage operation is indicated but as time goes on and experience becomes wider, we find fewer patients in this group who require the operation in stages, so that now it is a rare thing for us to do a ligation of the superior pole and almost as rare to remove the lobes one at a time.

2. Hyperthyroidism with crisis. Crisis occurring in severe hyperthyroidism is probably due to an acidosis. The markedly increased catabolism necessitates increased excretion and an increased dissipation of heat. The blood supply of the skin is increased in order to hasten the dissipation of heat through radiation and evaporation. The rapid evaporation reduces the fluids of the body concentrating the solids of the blood stream and making their absorption from the tissues more difficult. The increased blood supply to the skin means a relatively decreased blood supply to the kidneys, consequently, these patients are prone to develop an acidosis. Until we began to use glucose intravenously we had a limited number of patients each year coming in with an acute hyperthyroidism with vomiting and diarrhea which we were unable to control, and consequently, we were unable to give Lugol's solution because it was immediately expelled when given by either mouth or rectum. These patients usually died within two to four days after entering the hospital. Since we began to use glucose intravenously, I have not seen a single death from this cause. With the exception of patients brought in in a morabund condition this type of death should be extremely infrequent. It is our custom in this group to give patients I 000 c.c. of a Io per cent solution of glucose in physiologic salt solution intravenously as soon as they enter the hospital. This is given slowly, taking at Jeast an hour for administration and longer if there is evidence of myocardial weakening. Fifteen units of insulin are given at the beginning of the injection of the glucose and the same amount at the conclusion. The vomiting and diarrhea are rapidly checked and the patients are then able to take Lugol's solution by mouth. We prefer giving the Lugol's solution in orange juice or milk in 25 minim doses four times a day. After checking the crisis, the preparation of these patients does not differ from that of those with severe hyperthyroidism without crisis except that it usually takes a little longer to get them ready for operation.

3. Hyperthyroidism with cardiac fibrilIation. This group gives us more anxiety than any other group of goiter patients and yet our results are as satisfactory so far as operation is concerned. I have had only I death to be termed an operative death in this group and that in a woman seventy-nine years of age. Other patients have later died of heart lesions but this did not occur for some time after leaving the hospital with the exception of patient who died from emboli one month after leaving the hospital.

These patients are put at absolute rest in bed. Veronal and pantopon are used in sufficient dosage to keep them perfectly contented. Lugol's solution is given as to patients with severe hyperthyroidism. The heart condition has been treated by my associate, Dr. Luther T. NeIson, with quinidine or quinidine and digitalis. Some of the milder cases yield with this treatof the milder cases yield with this treat-
ment and the heart stops fibrillating, but in some of the more severe cases, the heart continues to fibrillate until the final subtotal Iobectomy.

It is in this group that the stage operation is most valuable. Upon those patients in whom the heart does not yield, the peration is done in four stages, ligating the superior poles one at a time and doing the subtotal lobectomies one at a time. I have many times observed that while thave many times observed that while the patient showed definite improvement after each of the stage operations, the heart continued to fibrillate until the removal of the last lobe. This is indicative of the extent of the operation required. Practically all the diseased thyroid tissue must be removed and new thyroid tissue developed in order to get a normal secretion. Even though the heart may appear to be normal, the patient should be warned that a badly damaged heart probably never becomes completely normal and consequently should never be severely taxed.

4. Hyperthyroidism with hypertension. Hypertension occurs in the course of hyperthyroidism from toxic adenoma or toxic diffuse adenomatous goiter and after repeated attacks of toxic hyperplastic (exophthaImic) goiter. Patients with pronounced hypertension have given me a great deal of anxiety for fear a cerebral hemorrhage would occur as a result of the operation. Up to the present, I have never seen it follow goiter operations, although I saw it only last year following a prostatectomy that was necessary in the presence of high hypertension because of marked urinary retention.

It is our rule never to operate in the presence of high hypertension except in case of necessity. I regard operation in toxic goiter with hypertension as a necessity because these patients gradually get worse. They are given bed rest with sedatives and Lugol's solution for one to two weeks previous to the operation. Ligations are rarely done, as the type of goiter producing hypertension usually does not give definite results from ligating the superior poles. Subtotal lobectomies are done in one or two stages according to the severity of the goiter and the height of the hypertension. Following the operation the hypertension usually is reduced but rarely returns to normal because of permanent damage done before operation.

5. Hyperthyroidism in the latter months of pregnancy. In the early months pregnancy does not constitute a contraindication for an operation for goiter. In fact, I have regarded it as an indication for an immediate operation because the patient with a mild hyperthyroidism may develop a very extreme hyperthyroidism in the course of the pregnancy. I have never seen an abortion occur as the result of the operation on a pregnant woman. In the latter months of pregnancy there is an added risk and I believe it is best to treat these patients with roentgen ray, telling them that it is a temporary measure. While it relieves the attack, it does not cure the goiter, which will recur later in a large proportion of the cases. It is my custom to give these patients Lugol's solution, the amount varying from Io minims three times a day to 25 minims four times a day for two weeks. Sedatives are given to control the nervous state of the patient and each lobe and the isthmus are given 90 milliampere-minutes of roentgen ray, 3 milliamperes of current for thirty minutes, with a 9 inch back-up, filtered through $6 \mathrm{~mm}$. of aluminum and $1 / 2$ inch of sole leather. This is repeated as indicated; usually only one treatment is given and the patient is advised to come in a month after delivery for the operation.

The operation done in severe cases must be radical. Recurrences and, what is more common, continuation of symptoms with only partial relief and which Iater become severe, are more common with incomplete operations upon this group than in the mild goiters. The entire thyroid gland is diseased in all except the isolated adenoma. The types I have been discussing are usually caused by the diffuse types of goiter. The patients belong to the desperate goiter group because of the severity of reaction to the secretion of a pathologic gland. A partial operation that leaves sufficient pathological gland to continue to produce the secretion does not cure. The operation must be so radical that were regeneration not to take place, myxedema must result because of a lack of secretion.

In experimental work I have demonstrated that regeneration with the development of normal thyroid tissue sufficient to meet the needs of the experimental 
animal will take place if the gland is that without the pre- and postoperative saturated with iodine and kept saturated saturation of the thyroid with iodine, for two months after the operation. In practice, I give to minims of Lugol's solution three times a day for one month after the operation and then once a day for the second month, and follow this with iodized salt or ro $\mathrm{mg}$. of iodine weekly in tablet form. I have never seen a recurrence when this method has been followed. Sometimes there will be a transitory hypothyroidism as a result of the radical operation but this is easily controlled with desiccated thyroid in small doses and has always cleared up, in patients I have seen, within one to three months.

Experimentally we have demonstrated excessive hyperplasia with true goiter development may occur.

In conclusion I wish to state that in my judgment, with careful study and careful handling before the operation, the desperate goiter patient does not offer a much greater risk than the milder case so far as the cure of the goiter itself is concerned. However, permanent lesions existing before the operation will still be present so that many patients will still have some of the effects of the severe goiter although the toxic goiter has been replaced by a thyroid gland with a perfectly normal secretion.

\section{연}

